\title{
Modeling of Milk Flow in Mammary Ducts in Lactating Human Female Breast
}

\author{
S. Negin Mortazavi \\ Graduate Student \\ Mechanical Engineering Department \\ University of Texas at Dallas \\ 800 West Campbell, Richardson, Texas, 75080 \\ Donna Geddes \\ Associate Professor \\ School of Chemistry and Biochemistry \\ University of Western Australia \\ Crawley WA, Australia \\ negin@utdallas.edu \\ Fatemeh Hassanipour \\ donna.geddes@uwa. edu. au \\ Assistant Professor \\ Mechanical Engineering Department \\ University of Texas at Dallas \\ 800 West Campbell, Richardson, Texas, 75080 \\ fatemeheutdallas.edu
}

\begin{abstract}
A transient laminar Newtonian three-dimensional CFD simulation has been studied for milk flow in a phantom model of the 6-generations human lactating breast branching system. Milk is extracted by the cyclic pattern of suction from the alveoli through the duct and to the nipple. The real negative (suction) pressure data are applied as an outlet boundary condition in nipple. In this study, the commercial CFD code (Fluent Inc., 2004) is employed for the numerical solution of the milk flow. The milk intake flow rate from simulation is compared to the real clinical data from published paper. The results are in good agreement. It is believed that the methodology of the lactating human breast branching modeling proposed here can provide potential guidelines for further clinical and research application.
\end{abstract}

\section{INTRODUCTION}

Flow in a bifurcating duct is one of the most important problems in the general case of organ physiology, e.g. the lung, the kidney, the breast, and other vascular tissue. A deeper knowledge of convective transport through bifurcation of such organs is essential for elucidating both the physical basis of the transport processes, and the pathology of many human diseases, such as agenesis and asthma in the kidney and lung, as well as breast carcinoma, a disease of terminal ductal lobular units [1]. Many aspects of the diagnosis, healing and investigation of the origin of breast diseases as well as various conventional breast conditions such as ductal blockage, breast engorgement, breast abscess, and galactocele which results in difficulties of breastfeeding would significantly benefit from a detailed understanding of the milk flow mechanism and related factors in removing milk from milk ducts to the nipple and infant mouth [2]. Despite the clear advantage of a fundamental understanding of the milk flow in human breast ductal system, this has not yet been the subject of careful computational fluid dynamic (CFD) modeling. Thus, the present study produces a simulation model providing new and interesting insights. Computational fluid dynamics (CFD) is a method that can be applied on 2D or $3 \mathrm{D}$ computer models to simulate the flow patterns using computational grid. This technique has been used in many fields of biomedical and biomechanical modeling in different organs. This paper aims to study the mechanism of removing milk by infant from the lactating breast. To reach this goal, the dynamic of infant suckling was well understood through the recent published study [3, 4]. The relationship between applied infant suckling pressure and milk yield is studied in current work. To investigate this relationship, the simulation model of the lactation branching ductal system is developed based on the phantom model. Our goal is to do a comparative study of the milk intake during infant suckling between clinical data and our simulation model. Therefore, current results are validated with a clinical experiment on 15 infants less than one month that were monitored during lactation and recordings of vacuum pressure and milk transfer rate were provided as a real experimental data [5].

\section{BREAST DUCTAL SYSTEM}

Branching ductal milk flow system in the lactating breast resembles a tree (Figure B.1). The branches of the tree are milk ducts and the leaves are the alveoli (tiny bulbs) which form spherical cavity structure and surround by epithelial cells. Milk is produced in alveoli from the water and nutrients that are diffused from the neighboring blood stream. There exist millions of alveoli in the lactating breast. A cluster of alveoli is named a lobule. Approximately 20 to 40 lobules are added to create a lobe [6]. More than 90Milk is circulated to different parts of the nipple from the alveoli through the ducts when baby is sucking. Since, the total ductal cross sectional area increases in each next branching ducts from nipple to alveoli, the milk flow is accelerated to reach the nipple. To the authors knowledge, the computational study described herein represents the first CFD simulation of the milk flow in branching ductal system of the breast. The main purpose of the present research is to provide a deeper understanding of the physiological aspects of the milk flow in milk ducts and to correlate the results with the classical clinical data and find a better use of extending CFD to clinical research. Because it has shown that collecting real data is time consuming 
TABLE I

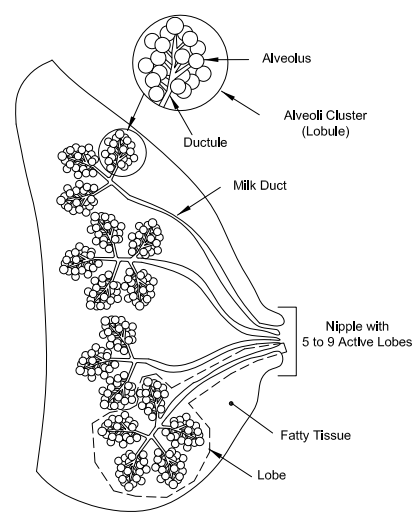

Pressure Profiles for OUtlet Boundary Condition

\begin{tabular}{|c|l|}
\hline Time $(\mathrm{s})$ & Pressure Profile $(\mathrm{kPa})$ \\
\hline $\mathrm{t}<20.44$ & $P(t)=0$ \\
\hline $20.44<\mathrm{t}<43.85$ & $P(t)=-26.3230 \sin ^{2}(3.4 t)-3.2744$ \\
\hline $43.85<\mathrm{t}<108.33$ & $P(t)=-16.4680 \sin ^{2}(3.2 t)-8.1167$ \\
\hline $108.33<\mathrm{t}<143.13$ & $P(t)=-1.1039$ \\
\hline $143.13<\mathrm{t}<190.45$ & $P(t)=-13.5200 \sin ^{2}(3.5 t)-9.2446$ \\
\hline $190.45<\mathrm{t}<193.96$ & $P(t)=-0.3773$ \\
\hline $193.96<\mathrm{t}<203.4800$ & $P(t)=-12.7430 \sin ^{2}(3.3 t)-11.7220$ \\
\hline $203.4800<\mathrm{t}<300.64$ & $P(t)=-12.2670 \sin ^{2}(1.9 t)-7.7221$ \\
\hline $300.64<\mathrm{t}<394.4300$ & $P(t)=-11.7910 \sin ^{2}(3.5 t)-3.7224$ \\
\hline $394.43<\mathrm{t}<399.19$ & $P(t)=-0.7466$ \\
\hline $399.19<\mathrm{t}<425.19$ & $\left.P(t)=-12.2720 \sin ^{2} 3.0 t\right)-4.8876$ \\
\hline $425.19<\mathrm{t}<428.08$ & $P(t)=-6.6181$ \\
\hline $428.08<\mathrm{t}<431.33$ & $P(t)=-13.0620 \sin ^{2}(4.4 t)-7.4861$ \\
\hline $431.33<\mathrm{t}<433.24$ & $P(t)=-8.4580$ \\
\hline $433.24<\mathrm{t}<445.34$ & $P(t)=-11.6100 \sin ^{2}(3.3 t)-5.8089$ \\
\hline $445.34<\mathrm{t}<449.96$ & $P(t)=-5.7262$ \\
\hline $449.96<\mathrm{t}<454.64$ & $P(t)=-14.5970 \sin ^{2}(3.5 t)-9.3699$ \\
\hline $454.64<\mathrm{t}<458.59$ & $P(t)=-0.4479 \sin ^{2}(43.1 t)-6.0048$ \\
\hline $458.59<\mathrm{t}<462.36$ & $P(t)=-118.8880 \sin ^{2}(3.6 t)-7.4141$ \\
\hline $462.36<\mathrm{t}<486.34$ & $P(t)=-0.2426 \sin ^{2}(15.7 t)-6.1782$ \\
\hline $486.34<\mathrm{t}<501.6300$ & $P(t)=-6.8728 \sin ^{2}(4.1 t)-5.0449$ \\
\hline
\end{tabular}

and difficult to relate specific parameters for milk flow rates, negative pressure profiles and many other factors to lactating breast, due to the high physiological variation of these parameters among women in different instances of breast feeding.

\section{COMPUTATIONAL SIMULATION}

\section{A. Geometry}

Designing the 3D model of lactating breast branching ductal system is one of the most challenging part of this research. In order to get more realistic results, the dimensions of the milk ducts and nipple as well as the number of generations and alveoli in model should be in good agreement with real breast data. Our first couple of attempts were using Ultrasound and MRI images of breast. While Ultrasound and MRI images of other biological organs like lung and kidney is clear enough to visualize the branching system, these types of images from breast are not clear in term of tracing the ducting paths(Figure C.2-a,b). The third attempt was done by using the branching ducts of breast model in the library of "Human Body Model" in ANSYS/ANSOFT(HFSS) software. The main problem of this model was related to the unrealistic shape of the duct cross sections which was formed in square, however the real cross sections are more close to circle(Figure C.2-c). The fourth option was using ductogram/galactogram images. These images are one special type of mammogram which use an x-ray dye injected into a duct for better visualization of ducts. By the means of MIMICS software these images can be reconstructed and used for computer simulation analysis. Since the ductograms are not imaged as a volume data set like a spiral CT, the final reconstructed model of these images in MIMICS would not be in 3D. So, it could not be helpful for the current modeling simulation(Figure C.2-d)

As the final option, we based our present CFD analysis on the published anatomical human breast model available to date; i.e. the phantom model which was designed using MilkShape 3D, a graphics model creation package. The model was based on a depiction derived from portrayals



Fig. C.3. Interior of breast phantom model using surface rendering techniques

in anatomy books. Neither MRI or CT scan images would provide the resolution and contrast to resolve the structures the way phantom model does. The main goal of designing breast phantom model was to support projects on breast simulation and imaging [8]. Figure C.3 portrays the interior of breast phantom using surface rendering techniques. Tissues include skin, areola, lobule, ductal, and blood. Considering the fact that breast include five to nine lobes, Figure C.4-Left shows a typical image of entire breast ductal system (in the solidwork software) by multiple copying of one lobe from phantom model. This ductal system has been used as the final geometry exported in the ANSYS-FLUENT software.

\section{B. Numerical Method}

The CFD simulation was performed for all milk duct branches of 6 generations of breast ductal system from alveoli to nipple. The tetrahedral unstructured meshes were used for the interior of the milk dutcs with total number of elements of about $2.52 \times 10^{6}$ (Figure C.4-Right). The problem was first solved with a fairly coarse mesh. After convergence, the solution was improved by refining the mesh 


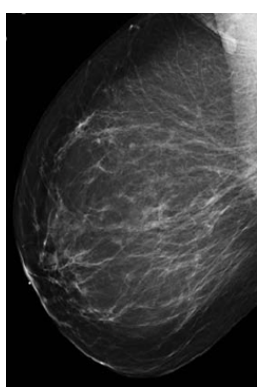

(a)



(b)

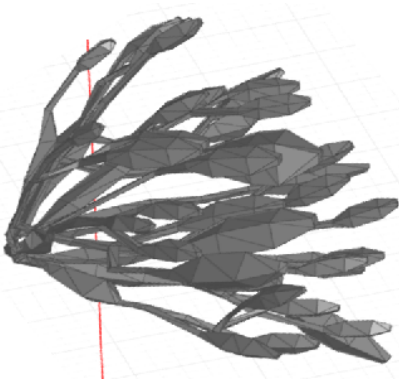

(c)

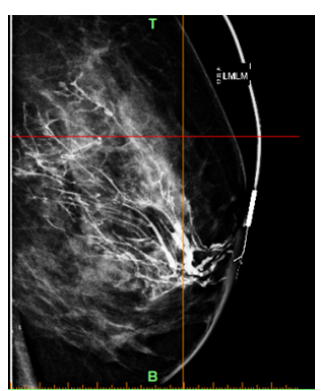

(d)

Fig. C.2. Sample (a) Ultrasound, (b) MRI, (c) Human body library, and (d) Ductogram images of breast

(gradient adaption tool [9]).

In this study, the commercial CFD code (Fluent Inc., 2004) was employed for the numerical solution of the milk flow from alveoli through milk ducts and to the nipple. All transport equations were discretized by second-order accurate time stepping and a second order accurate discretization scheme. The SIMPLE algorithm was applied to evaluate the pressure-velocity coupling. The laminar solution of the flow field was assumed to have converged when the continuity and momentum equations residuals reduced to less than 0.000001. Time step independence was required for all simulations specially for transient problems. Table II represents results for milk flow rate, show that the optimum time step of 0.0625 seconds is proper value for running the simulation. the The number of iterations per time steps were assigned high enough to provide the convergence criterion condition.

The milk was considered as an incompressible Newtonian fluid with the density of $1030\left(\mathrm{~kg} / \mathrm{m}^{3}\right)$, and the viscosity of $(1.66-51.9) \times 10^{-6} \mathrm{~m}^{2} / \mathrm{s}$. The large range of viscosity is due to the variation of viscosity in different women. The gravitational effect was neglected. In this study, the milk flow motion in ducts was assumed as unsteady laminar flow and described by the momentum conservation (Navier-Stokes equations) and mass conservation (continuity equation) equations.

\section{Boundary Condition}

To simulate the transient model of milk flow, the axial cyclic negative pressure (suction pressure) is imposed across the nipple as an outlet pressure (Table I). The periodic pressure profile was written in separate user defined functions (UDF) in $\mathrm{C}$ language and interpreted in Fluent. These pressure profiles are based on the real clinical data. While the suckling pressures are applied to the nipple side of the lobe, the proximal end (fixed position adjacent to alveoli) is considered to have zero pressure. Considering the fact that infant suckling is dominant to the minor peripheral pressures by duct tissue, for simplicity the walls of the milk ducts are considered as rigid wall with no-slip boundary condition. Both inlet and outlet boundary conditions are applied perpendicular to the inlet (alveoli) and outlet (nipple) cross sections.
TABLE II

REPRESENTATIVE TIME STEP ACCURACY

\begin{tabular}{|c|c|}
\hline Time step(s) & Milk flow rate $(\mathrm{kg} / \mathrm{s})$ \\
\hline 0.5 & 0.0000638 \\
\hline 0.25 & 0.0000406 \\
\hline 0.125 & 0.000019 \\
\hline 0.0625 & 0.000017 \\
\hline 0.03125 & 0.0000166 \\
\hline
\end{tabular}
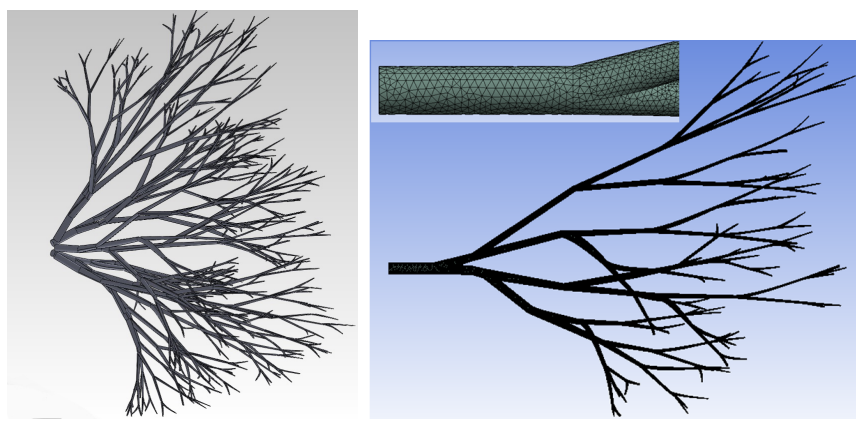

Fig. C.4. (Left): 3D anterior visualization of breast ductal branching model with 5 lobes (Right): Anterior view of generated mesh of 6 generations of breast ductal system with $2.52 \times 10^{6}$ elements

\section{RESULT}

The recorded intra-oral vacuum pressure from the clinical experiments is used in simulation as an outlet boundary condition [5]. Vacuum pressure is not applied uniformly with the same period and number of sucks for the entire breastfeeding duration, but rather changes with the infant's patterns sucking defined by the presence or absence of the milk. The role of this negative suction pressure is to help refilling the nipple by milk from the ducts, and keeping the nipple and breast in correct position inside the infant's mouth.

The following equation shows the general format of the periodic outlet pressure profile (Table I):

$$
p(t)=A \sin ^{2}(\xi t)+B
$$

Where $\mathrm{A}$ is the difference between baseline and peak pressure, $\mathrm{B}$ is the peak pressure and $\xi$ is the frequency of the pressure profile.

Applying pressure profiles (Table I) in CFD simulation and running for a breastfeeding time duration, the pressure 
TABLE III

RESULTS FOR AVERAGE MILK FLOW RATE BY CLINICAL EXPERIMENT ON 15 INFANTS[5]

\begin{tabular}{|c|c|c|}
\hline Milk intake(g) & Feed duration(s) & Derived milk flow rate \\
\hline $93.1 \pm 32.4$ & $869.6 \pm 627.9$ & 0.0001070 \\
\hline
\end{tabular}

contours are derived. Figure D.5 shows three different pressure contours for minimum, average and maximum value of pressures in the ductal system respectively. In this work, milk flow in one lobe is simulated. Since each lactating breast has 5-9 lobes, the total milk intake and therefore the total mass flow rate should times by the total number of lobes. So, the derived mass flow rate for full breast is equal to 0.000085 $(\mathrm{kg} / \mathrm{s})$ [11]. It is not our aim to develop an intricate model which incorporates the complicated structure of the lactating ductal breast. Instead, our goal is to create a conservative model which contains the important mechanisms related to the removing of milk. However we make many assumptions, our model is appropriate in validating the results with the real clinical data $[5,10]$. The measured milk intake $(\mathrm{g})$ and breastfeeding duration(s) in experimental analysis by the author are recorded in Table III. Milk flow rate is calculated by the ratio of milk intake and breastfeeding duration. As shown in table, the milk flow rate in our simulation is in good agreement with the experimental results.

\section{CONCLUSION}

In this work we have done the CFD simulation of the milk flow in branching ductal system of the human lactating breast. The 3D geometry is based on the phantom model of the breast [Baum 2008].

The main purpose of the research is to provide a better understanding of the anatomy of the milk flow in milk ducts and do a comparative study of the milk intake during infant suckling between clinical data and our simulation model. The simulation results are in good agreement with the published experimental data.

This research also demonstrates the benefits of using CFD. Collecting real data is time consuming and difficult to relate specific parameters for milk flow rates, negative pressure profiles and many other factors to lactating breast, due to the high physiological variation of these parameters among women in different instances of breast feeding. Therefore, extending CFD to clinical research has shown several advantages.

\section{ACKNOWLEDGMENT}

The authors would like to acknowledge Dr. Karl G Baum(Biomedical and Materials Multimodal Imaging Laboratory, Rochester Institute of Technology, Chester F. Carlson Center for Imaging Science) for 3D phantom model of the human breast.

\section{REFERENCES}

[1] Love, S. M., Barsky, S. H., Anatomy of the nipple and breast ducts revisited, Cancer, 1947, vol. 101(9), pp. 1947-1957.

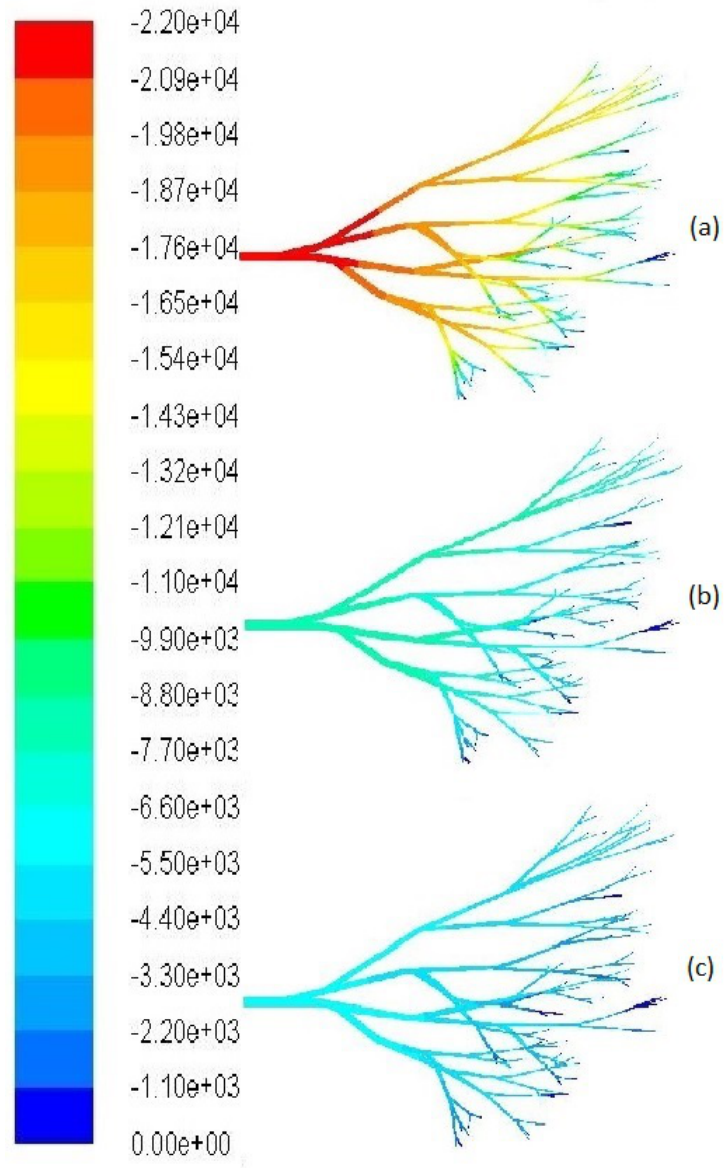

Fig. D.5. Contours of pressure at (a) $t=300 s$, (b) $t=200 s,(c) t=150 \mathrm{~s}$

[2] Lu, P., Sternlicht, M. D., Werb, Z., Comparative mechanisms of branching morphogenesis in diverse systems, Journal of mammary gland biology and neoplasia, 2006, vol. 11(3-4), pp. 213-228. .

[3] Zoppou, C., Barry, S., Mercer, G., Dynamics of human milk extraction: A comparative study of breast feeding and breast pumping, Bulletin of mathematical biology, 1997, vol.59(5), pp. 953-973.

[4] Ramsay, D. T., Mitoulas, L. R., Kent, J. C., Cregan, M. D., Doherty, D. A., Larsson, M., Hartmann, P. E., Milk flow rates can be used to identify and investigate milk ejection in women expressing breast milk using an electric breast pump, Breastfeeding Medicine, 2006, vol. 1(1), pp. 14-23.

[5] Sakalidis, V. S., Kent, J. C., Garbin, C. P., Hepworth, A. R., Hartmann, P. E., Geddes, D. T., Longitudinal changes in suck-swallow-breathe, oxygen saturation, and heart rate patterns in term breastfeeding infants, Journal of Human Lactation, 2013, vol.29 (2), pp. 236-245.

[6] Sohn, C., Blohmer, J.U., Hamper, U.M., Breast ultrasound: a systematic approach to technique and image interpretation, Thieme, 1999.

[7] Ramsay, D., Kent, J., Hartmann, R., Hartmann, P., Anatomy of the lactating human breast redefined with ultrasound imaging, Journal of Anatomy, 2005,vol.206 (6), pp.525-534.

[8] Baum, K. G., 2008. Multimodal breast imaging: Registration, visualization, and image synthesis. ProQuest.

[9] Fluent, A., Ansys fluent 12.0 tutorial guide. Ansys INC, 2009.

[10] Geddes, D. T., Sakalidis, V. S., Hepworth, A. R., McClellan, H. L., Kent, J. C., Lai, C. T., Hartmann, P. E., Tongue movement and intraoral vacuum of term infants during breastfeeding and feeding from an experimental teat that released milk under vacuum only, Early human development, 2012,vol.88 (6), pp.443-449.

[11] Mortazavi, N. and Hassanipour, F., Computational Fluid Dymnamic Modeling of Milk in Mammary Ducts in Lactating Human Females, The Seventh International Conference on Thermal Engineering Theory and Applications, Marrakesh, Morocco, May 2014. 